\title{
ON THE CONDITION NUMBER OF INTEGRAL EQUATIONS IN LINEAR ELASTICITY USING THE MODIFIED GREEN'S FUNCTION
}

\author{
E. ARGYROPOULOS ${ }^{1}$, D. GINTIDES ${ }^{1}$ and K. KIRIAKI ${ }^{1}$
}

(Received 14 August, 1999; revised 29 December, 2000)

\begin{abstract}
In this work the modified Green's function technique for an exterior Dirichlet and Neumann problem in linear elasticity is investigated. We introduce a modification of the fundamental solution in order to remove the lack of uniqueness for the solution of the boundary integral equations describing the problems, and to simultaneously minimise their condition number. In view of this procedure the cases of the sphere and perturbations of the sphere are examined. Numerical results that demonstrate the effect of increasing the number of coefficients in the modification on the optimal condition number are also presented.
\end{abstract}

\section{Introduction}

As is well-known, the reformulation of an exterior boundary value problem to a boundary integral equation presents difficulties caused by the lack of uniqueness of its solutions. In order to remove this problem the modified Green's function technique was proposed by Jones [8] and Ursell [13] for the acoustical case. In [10, 11] Kleinman and Roach have shown that the choice of coefficients of the modification, apart from the removal of the non-uniqueness problem, can also satisfy other criteria of best modification. These include that of the best approximation to the actual Green's function and that of minimisation of the norm of the modified integral operator. In [9] Kleinman and Kress have established the criterion of minimisation of the condition number of the boundary integral equation for the acoustical case.

Similar arguments hold for the elastic case. The first work in linear elasticity in which this technique was introduced is due to Jones [8]. In [4,5] results for the elastic two-dimensional case are presented. In [1,2] and [7] exterior elastic problems in $R^{3}$ are examined and criteria for best modification are also established.

\footnotetext{
${ }^{1}$ Department of Mathematics, National Technical University of Athens, Zografou Campus, 15780 Athens, Greece; e-mail: kkouli@math.ntua.gr.

(C) Australian Mathematical Society 2003, Serial-fee code 1446-1811/03
} 
In this work the modified Green's function technique is adopted and, using it, the minimisation of the condition number of the boundary integral equations describing the exterior Dirichlet and Neumann problems is established.

Many ideas of [9] are exploited, nevertheless there are noteworthy differences between the acoustic and the elastic case. In Section 2 the formulation of the problems in integral form through a layer theoretic approach is given. In Section 3 the main idea of the Green's function technique is presented. The free-space Green's function and the regular part of the modification are expressed in Hansen eigenvectors. In Section 4 the criterion of minimisation is established for the spherical rigid body and the cavity. In Section 5 the shapes which can be produced as perturbations of the sphere are discussed. Finally in Section 6 numerical implementation of the whole technique is given. The figures in this section demonstrate the effect of adding non-vanishing terms to the modified Green's function. The nature of the problem in elasticity admits the presence of two different types of eigenvalues: spheroidal and toroidal. These eigenvalues are removed by adding different types of terms in the modification.

The need for adopting minimisation criteria in elasticity relies on the fact that the finally derived linear systems incorporate large amounts of error stemming from approximations of surface integrations over weakly and strongly singular kernels, standard numerical errors, etc. Therefore it is extremely useful to implement methods which ensure stability. From this point of view, each technique for choosing the modification coefficients constitutes a strategy to solve numerically complicated elasticity problems. The method of minimising the norm of the operator is appropriate for operator approximation schemes such as, for example, Neumann operator series. The method which is presented in this paper is proper for direct inversion of the approximate linear systems using, for example, iteration schemes. The case of the sphere is quite interesting because analytical and numerical estimations reveal the main characteristics of the modified Green's function method. These results can be applied to perturbations of the sphere and potentially can be applied in more general boundaries. For spheres, the criterion of minimisation of the operator norm gives results which can be recovered from the general choice of coefficients provided by the method of minimising the condition number, but this result is limited to this geometry where, as proved in [1], it corresponds to a degenerate integral operator.

\section{Statement of the problem}

Let $D_{-}$denote a bounded connected domain in $R^{3}$ with boundary $\partial D$, which will be assumed closed, bounded and Lyapunov. Let $D_{+}=R^{3} / \bar{D}_{-}$, where $\bar{D}_{-}=D_{-} \cup \partial D$. We assume that $D_{+}$is filled by an isotropic and homogeneous elastic medium specified by the Lamé constants $\lambda, \mu$ and mass density $\rho$. If the harmonic time dependence 
$e^{-i \omega t}$ is suppressed, then the displacement field $\mathbf{u}(\mathbf{r})$, in the absence of body forces, satisfies the time-reduced Navier equation of linearised elasticity

$$
\left(\Delta^{*}+\omega^{2} \rho\right) \mathbf{u}(\mathbf{r})=\mathbf{0},
$$

where the Kupradze operator $\Delta^{*}$ is given by the relation $\Delta^{*}=: \mu \Delta+(\lambda+\mu) \nabla \nabla$. and $\omega$ is the angular frequency. It is well-known that the solution of (2.1) is the sum of a longitudinal wave $\mathbf{u}_{p}$ propagating with phase velocity $c_{p}$ and a transverse wave $\mathbf{u}_{s}$ propagating with phase velocity $c_{s}$, that is, $\mathbf{u}(\mathbf{r})=\mathbf{u}_{p}(\mathbf{r})+\mathbf{u}_{s}(\mathbf{r})$. We also define the surface stress operator

$$
T=2 \mu \hat{\eta} \cdot \nabla+\lambda \hat{\eta} \nabla \cdot+\mu \hat{\eta} \times \nabla \times,
$$

where $\hat{\eta}$ is the exterior unit normal on $\partial D$. The radiation conditions, due to Kupradze, which the longitudinal and the transverse components of the displacement field must satisfy at the radiation zone are [12]

$$
\begin{aligned}
r\left\{\frac{\partial \mathbf{u}_{a}(\mathbf{r})}{\partial r}-i k_{a} \mathbf{u}_{a}(\mathbf{r})\right\} & =o(1), \quad r=|\mathbf{r}| \rightarrow \infty, \\
\mathbf{u}_{a}(\mathbf{r}) & =o(1),
\end{aligned}
$$

where $a=p$ and $s$ and $k_{p}^{2}=\rho \omega^{2} /(\lambda+2 \mu), k_{s}^{2}=\rho \omega^{2} / \mu$ are the wavenumbers for the longitudinal and the transverse waves respectively. The above conditions are required to hold for all directions of $\mathbf{r}$ uniformly.

The exterior boundary value problems which we examine are the problems of the rigid body and the cavity. So we have to determine the displacement field which satisfies the differential equation (2.1) for $D_{+}$, the boundary conditions

$$
\mathbf{u}(\mathbf{r})=\mathbf{f}(\mathbf{r}), \quad \mathbf{r} \in \partial D
$$

or

$$
T \mathbf{u}(\mathbf{r})=\mathbf{g}(\mathbf{r}), \quad \mathbf{r} \in \partial D
$$

where $\mathbf{f}, \mathbf{g}$ are known functions, for the Dirichlet and the Neumann problem respectively, and the radiation conditions (2.2).

In order to reformulate the problem in integral form, we can follow either the direct method, based on Betti's formulae, or the indirect method using the layer potentials. Following the layer theoretic approach, we define the single layer potential

$$
(S \varphi)(\mathbf{r})=\frac{1}{2 \pi} \int_{\partial D} \widetilde{\Gamma}\left(\mathbf{r}, \mathbf{r}^{\prime}\right) \cdot \varphi\left(\mathbf{r}^{\prime}\right) d S\left(\mathbf{r}^{\prime}\right)
$$

for a density $\varphi \in L_{2}(\partial D)$ and the double layer potential

$$
(D \varphi)(\mathbf{r})=\frac{1}{2 \pi} \int_{\partial D} \varphi\left(\mathbf{r}^{\prime}\right) \cdot T^{\left(\mathbf{r}^{\prime}\right)} \widetilde{\boldsymbol{\Gamma}}\left(\mathbf{r}, \mathbf{r}^{\prime}\right) d S\left(\mathbf{r}^{\prime}\right),
$$


where $\widetilde{\boldsymbol{\Gamma}}\left(\mathbf{r}, \mathbf{r}^{\prime}\right)$ is the free-space fundamental dyadic in $R^{3}$ which satisfies the equation

$$
\left(\Delta^{*}+\omega^{2} \rho\right) \widetilde{\boldsymbol{\Gamma}}\left(\mathbf{r}, \mathbf{r}^{\prime}\right)=-4 \pi \tilde{I} \delta\left(\mathbf{r}-\mathbf{r}^{\prime}\right)
$$

Here $\tilde{I}$ denotes the identity dyadic and $\widetilde{\Gamma}\left(\mathbf{r}, \mathbf{r}^{\prime}\right)$ is given by the relation

$$
\widetilde{\Gamma}\left(\mathbf{r}, \mathbf{r}^{\prime}\right)=\frac{1}{\mu} \frac{e^{i k_{s}\left|\mathbf{r}-\mathbf{r}^{\prime}\right|}}{\left|\mathbf{r}-\mathbf{r}^{\prime}\right|} \tilde{I}-\frac{1}{\mu k_{s}^{2}} \nabla_{\mathbf{r}} \otimes \nabla_{\mathbf{r}}\left(\frac{e^{i k_{p}\left|\mathbf{r}-\mathbf{r}^{\prime}\right|}}{\left|\mathbf{r}-\mathbf{r}^{\prime}\right|}-\frac{e^{i k_{s}\left|\mathbf{r}-\mathbf{r}^{\prime}\right|}}{\left|\mathbf{r}-\mathbf{r}^{\prime}\right|}\right)
$$

The subscripts and the superscripts in the above relations indicate that the action of the differential operators is on the indicated variable.

Exploiting the jump relations at the boundary [12], we can see that if we seek solutions of the exterior Dirichlet problem in terms of a double layer potential of an unknown density $\varphi$, then $\varphi$ is required to satisfy the boundary integral equation

$$
\left(I+\bar{K}^{*}\right) \varphi(\mathbf{r})=\mathbf{f}(\mathbf{r}), \quad \mathbf{r} \in \partial D
$$

where the integral operator appearing in (2.5) is the $L_{2}$-adjoint of $K$ given by the relation

So $K^{*}$ may be expressed as

$$
(K \varphi)(\mathbf{r})=\frac{1}{2 \pi} \int_{\partial D} \varphi\left(\mathbf{r}^{\prime}\right) \cdot T^{(\mathbf{r})} \widetilde{\mathbf{\Gamma}}\left(\mathbf{r}, \mathbf{r}^{\prime}\right) d S\left(\mathbf{r}^{\prime}\right) .
$$

$$
\left(K^{*} \varphi\right)(\mathbf{r})=\frac{1}{2 \pi} \int_{\partial D} \varphi\left(\mathbf{r}^{\prime}\right) \cdot \overline{T^{(\mathbf{r})} \tilde{\mathbf{\Gamma}}\left(\mathbf{r}, \mathbf{r}^{\prime}\right)} d S\left(\mathbf{r}^{\prime}\right)
$$

and the bar in (2.5) indicates the complex conjugate. Similarly the solution of the exterior Neumann problem may be expressed as a single layer potential of an unknown density which satisfies the integral equation

$$
(I-K) \varphi(\mathbf{r})=-\mathbf{g}(\mathbf{r}), \quad \mathbf{r} \in \partial D
$$

The above defined integral operators $K$ and $K^{*}$ have singular kernels. In [12] a Fredholm-type theory for these boundary integral equations based on a regularisation procedure is established. The global regulariser, which is shown to exist, is equivalent. So the original and the regularised equations have the same solutions. Many properties of the resolvents are also presented. It is proven that the homogeneous interior Dirichlet or Neumann problem has a discrete spectrum. In order to have uniqueness for the boundary integral equation describing the exterior problem we have to avoid the irregular frequencies, that is, the eigenvalues of the adjoint interior problem. To accommodate this difficulty we shall adopt the modified Green's function technique. 


\section{The modified Green's function technique}

As in [2] we assume that the modified part $\widetilde{U}\left(\mathbf{r}, \mathbf{r}^{\prime}\right)$ is a solution of the Lamé equation (2.1) which is regular in both variables $\mathbf{r}, \mathbf{r}^{\prime}$. So the dyadic

$$
\Gamma_{1}\left(\mathbf{r}, \mathbf{r}^{\prime}\right)=\widetilde{\Gamma}\left(\mathbf{r}, \mathbf{r}^{\prime}\right)+\widetilde{U}\left(\mathbf{r}, \mathbf{r}^{\prime}\right),
$$

where $\widetilde{\Gamma}\left(\mathbf{r}, \mathbf{r}^{\prime}\right)$ is the free-space fundamental solution given by (2.4), is also a solution of (2.3). Using this as a kernel we can also define single and double layer potentials and in view of modified jump relations, we can produce modified boundary integral equations.

The main motivation for the modification of the Green's function is an appropriate choice of the regular part in (3.1), so that it is possible to restore uniqueness of the solutions of the boundary integral equations and simultaneously achieve other desirable properties.

If we expand the modified fundamental solution in terms of a known eigenvector system, the problem is to find suitable coefficients for the expansion of the regular part. So we use the Hansen eigenvectors given by the relations

$$
\begin{aligned}
\mathbf{M}_{m n}^{e, i}(\mathbf{r})= & \sqrt{n(n+1)} f_{n}^{e, i}\left(k_{s} r\right) \mathbf{C}_{m n}(\theta, \phi), \\
\mathbf{N}_{m n}^{e, i}(\mathbf{r})= & \sqrt{n(n+1)}\left[\frac{f_{n}^{e, i}\left(k_{s} r\right)}{k_{s} r} \mathbf{P}_{m n}(\theta, \phi)\right. \\
& \left.+\left(f_{n}^{e, i \prime}\left(k_{s} r\right)+\frac{f_{n}^{e, i}\left(k_{s} r\right)}{k_{s} r}\right) \mathbf{B}_{m n}(\theta, \phi)\right], \\
\mathbf{L}_{m n}^{e, i}(\mathbf{r})= & f_{n}^{e, i \prime}\left(k_{p} r\right) \mathbf{P}_{m n}(\theta, \phi)+\sqrt{n(n+1)} \frac{f_{n}^{e, i}\left(k_{p} r\right)}{k_{p} r} \mathbf{B}_{m n}(\theta, \phi),
\end{aligned}
$$

where $n=0,1,2, \ldots,|m| \leq n$ and the superscripts $e, i$ stand for exterior and interior solutions respectively. Here $f_{n}^{e, i}$ are the spherical Bessel functions $f_{n}^{i}=j_{n}$, $f_{n}^{e}=h_{n}^{(1)}$, and the prime denotes differentiation with respect to the argument. We note that $\mathbf{P}_{m n}(\theta, \phi), \mathbf{B}_{m n}(\theta, \phi)$ and $\mathbf{C}_{m n}(\theta, \phi)$ in (3.2)-(3.4) are the well-known mutually orthogonal surface vector harmonics [3], which are given by the relations

$$
\begin{aligned}
\mathbf{P}_{m n}(\theta, \phi) & :=\hat{\mathbf{r}} Y_{n}^{m}(\theta, \phi), \\
\sqrt{n(n+1)} \mathbf{B}_{m n}(\theta, \phi) & :=\left(\hat{\boldsymbol{\theta}} \frac{\partial}{\partial \theta}+\hat{\boldsymbol{\phi}} \frac{1}{\sin \theta} \frac{\partial}{\partial \phi}\right) Y_{n}^{m}(\theta, \phi), \\
\sqrt{n(n+1)} \mathbf{C}_{m n}(\theta, \phi) & :=\left(\hat{\boldsymbol{\theta}} \frac{1}{\sin \theta} \frac{\partial}{\partial \phi}-\hat{\boldsymbol{\phi}} \frac{\partial}{\partial \theta}\right) Y_{n}^{m}(\theta, \phi),
\end{aligned}
$$

where the spherical harmonics are

$$
Y_{n}^{m}(\theta, \phi)=\sqrt{(2 n+1) \frac{(n-m) !}{(n+m) !}} P_{n}^{m}(\cos \theta) e^{i m \phi}
$$


and $\hat{\mathbf{r}}, \hat{\boldsymbol{\theta}}$ and $\hat{\boldsymbol{\phi}}$ are the unit vectors of the spherical coordinates.

In view of this eigenvector system the fundamental dyadic solution admits the representation [3]

$$
\begin{aligned}
\widetilde{\Gamma}\left(\mathbf{r}, \mathbf{r}^{\prime}\right)= & \frac{i k_{s}}{\mu} \sum_{n=1,1,0}^{\infty} \sum_{m=-n}^{n} \frac{1}{n(n+1)}\left[\mathbf{M}_{m n}^{-\epsilon}(\mathbf{r}) \otimes \dot{\mathbf{M}}_{m n}^{*}\left(\mathbf{r}^{\prime}\right)\right. \\
& \left.+\mathbf{N}_{m n}^{-\epsilon}(\mathbf{r}) \otimes \stackrel{\mathbf{N}}{\epsilon n}^{\epsilon}\left(\mathbf{r}^{\prime}\right)+n(n+1)\left(\frac{k_{p}}{k_{s}}\right)^{3} \mathbf{L}_{m n}^{-\epsilon}(\mathbf{r}) \otimes \dot{\mathbf{L}}_{m n}^{\epsilon}\left(\mathbf{r}^{\prime}\right)\right],
\end{aligned}
$$

where $\epsilon=\operatorname{sign}\left(r-r^{\prime}\right), r=|\mathbf{r}|, r^{\prime}=\left|\mathbf{r}^{\prime}\right|$. When $\epsilon=(+),(-)$ this corresponds to superscript ( $i$ ), (e) respectively. The summation over $n$ for $\mathbf{M}_{m n}$ and $\mathbf{N}_{m n}$ starts from $n=1$, while for $\mathbf{L}_{m n}$ it starts from $n=0$. The starred quantities are obtained by changing $e^{i m \phi}$ into $e^{-i m \phi}$. Similarly the eigenvector expansion of $\widetilde{U}\left(\mathbf{r}, \mathbf{r}^{\prime}\right)$, the regular part of the modification, is

$$
\begin{aligned}
\widetilde{U}\left(\mathbf{r}, \mathbf{r}^{\prime}\right)= & \frac{i k_{s}}{\mu} \sum_{n=1.1 .0}^{\infty} \sum_{m=-n}^{n} \frac{1}{n(n+1)}\left[a_{m n}^{1} \mathbf{M}_{m n}^{e}(\mathbf{r}) \otimes \stackrel{\mathbf{M}}{m n}^{e}\left(\mathbf{r}^{\prime}\right)\right. \\
& +a_{m n}^{2} \mathbf{N}_{m n}^{e}(\mathbf{r}) \otimes \stackrel{\mathbf{N}}{\mathbf{N}}_{m n}^{e}\left(\mathbf{r}^{\prime}\right)+n(n+1)\left(\frac{k_{p}}{k_{s}}\right)^{3} a_{m n}^{3} \mathbf{L}_{m n}^{e}(\mathbf{r}) \otimes \stackrel{\mathbf{L}}{m n}_{m}^{e}\left(\mathbf{r}^{\prime}\right) \\
& \left.+a_{m n}^{4} \mathbf{N}_{m n}^{e}(\mathbf{r}) \otimes \stackrel{\mathbf{L}_{m n}^{e}}{e}\left(\mathbf{r}^{\prime}\right)+n(n+1)\left(\frac{k_{p}}{k_{s}}\right)^{3} a_{m n}^{5} \mathbf{L}_{m n}^{e}(\mathbf{r}) \otimes \stackrel{\mathbf{N}}{m}_{m}^{e}\left(\mathbf{r}^{\prime}\right)\right]
\end{aligned}
$$

As is clarified in [2] the presence of the last two terms in the sum of (3.8), which we call "cross terms", is necessary in order to examine the special case of the sphere. In $[2,7]$ sufficient conditions on the coefficients of the modification for unique solvability of the boundary integral equation are established. In [1] we have also proposed a criterion for minimising the norm of the modified integral operator.

\section{On the condition number: the case of the sphere}

If we introduce the operators $M, N: L_{2}(\partial D) \rightarrow L_{2}(\partial D)$, where $M:=I+\bar{K}_{1}^{*}$, $N:=I-K_{1}$ then (2.5) and (2.6) may be written as

$$
M \phi=\mathbf{f}, \quad N \phi=-\mathbf{g} .
$$

We also introduce their $L_{2}$-adjoints $M^{*}$ and $N^{*}$.

As is well-known, the condition number which is given by the relation [6]

$$
\operatorname{cond}(M):=\|M\|\left\|M^{-1}\right\|
$$


with respect to the $L_{2}$-norm can be expressed as cond $(M)=\left(\lambda_{\max }^{M} / \lambda_{\min }^{M}\right)^{1 / 2}$, where $\lambda_{\max }^{M}$ and $\lambda_{\min }^{M}$ denote the largest and the smallest spectral value of the self-adjoint operator $M^{*} M$. The condition number of the operator $N$ is similarly given in terms of the largest and the smallest spectral values of $N^{*} N$.

As is shown in [11], it is extremely difficult to get explicit results for the coefficients which minimise the operator norms for arbitrary boundaries $\partial D$. Similar discussion is given in [9] for the condition number of integral equations in acoustics, using modified Green's functions. Nevertheless, the special result for minimising the condition number when $\partial D$ is a sphere serves as a guide to an explicit coefficient choice which leads to well conditioned integral equations for perturbations of spherical domains. So we examine first the spherical rigid body and the spherical cavity. It is easily proved that the following relations hold for a sphere centered at the origin with radius $R$ :

$$
T^{(\mathbf{r})} \widetilde{\Gamma}\left(\mathbf{r}, \mathbf{r}^{\prime}\right)=\left[T^{\left(r^{\prime}\right)} \widetilde{\Gamma}\left(\mathbf{r}, \mathbf{r}^{\prime}\right)\right]^{T} \quad \text { and } \quad T^{(r)} \widetilde{U}\left(\mathbf{r}, \mathbf{r}^{\prime}\right)=\left[T^{\left(r^{\prime}\right)} \widetilde{U}\left(\mathbf{r}, \mathbf{r}^{\prime}\right)\right]^{T} \text {, }
$$

where the superscript " $T$ " indicates the transpose matrix. In view of (4.1) we conclude that $K_{1}=\bar{K}_{1}^{*}$. We now consider the modified single layer potentials with densities given by the vector spherical harmonics $\mathbf{P}_{m n}, \mathbf{B}_{m n}, \mathbf{C}_{m n}$ :

$$
\begin{aligned}
& \mathbf{V}_{m n}^{P}(\mathbf{r})=\frac{1}{2 \pi} \int_{\partial D_{R}} \widetilde{\Gamma}_{1}\left(\mathbf{r}, \mathbf{r}^{\prime}\right) \cdot \mathbf{P}_{m n}(\theta, \phi) d S\left(\mathbf{r}^{\prime}\right), \\
& \mathbf{V}_{m n}^{B}(\mathbf{r})=\frac{1}{2 \pi} \int_{\partial D_{R}} \widetilde{\Gamma}_{1}\left(\mathbf{r}, \mathbf{r}^{\prime}\right) \cdot \mathbf{B}_{m n}(\theta, \phi) d S\left(\mathbf{r}^{\prime}\right), \\
& \mathbf{V}_{m n}^{c}(\mathbf{r})=\frac{1}{2 \pi} \int_{\partial D_{R}} \widetilde{\Gamma}_{1}\left(\mathbf{r}, \mathbf{r}^{\prime}\right) \cdot \mathbf{C}_{m n}(\theta, \phi) d S\left(\mathbf{r}^{\prime}\right) .
\end{aligned}
$$

Substituting the expressions (3.5)-(3.6) in (4.2)-(4.4) and exploiting the orthogonality relations for the surface vector harmonics we obtain the relations

$$
\begin{aligned}
\mathbf{V}_{m n}^{P}(\mathbf{r})= & \frac{2 i k_{s}}{\mu}\left[\left(\mathbf{N}_{m n}^{i}(\mathbf{r})+a_{m n}^{2} \mathbf{N}_{m n}^{e}(\mathbf{r})+\frac{a_{m n}^{4}}{n(n+1)} \frac{f_{n}^{e \prime}\left(k_{p} r\right) k_{s} r}{f_{n}^{e}\left(k_{s} r\right)} \mathbf{N}_{m n}^{e}(\mathbf{r})\right) \frac{f_{n}^{e}\left(k_{s} r\right)}{k_{s} r}\right. \\
& \left.+\left(\frac{k_{p}}{k_{s}}\right)^{3}\left(\mathbf{L}_{m n}^{i}(\mathbf{r})+a_{m n}^{3} \mathbf{L}_{m n}^{e}(\mathbf{r})+\frac{a_{m n}^{5} f_{n}^{e}\left(k_{s} r\right)}{f_{n}^{e \prime}\left(k_{p} r\right) k_{s} r} \mathbf{L}_{m n}^{e}(\mathbf{r})\right) f_{n}^{e \prime}\left(k_{p} r\right)\right] R^{2} \\
\mathbf{V}_{m n}^{B}(\mathbf{r})= & \frac{2 i k_{s}}{\mu \sqrt{n(n+1)}}\left[\left(\mathbf{N}_{m n}^{i}(\mathbf{r})+a_{m n}^{2} \mathbf{N}_{m n}^{e}(\mathbf{r})\right.\right. \\
& \left.+a_{m n}^{s} \mathbf{L}_{m n}^{e}(\mathbf{r})\right)\left(f_{n}^{e \prime}\left(k_{s} r\right)+\frac{f_{n}^{e}\left(k_{s} r\right)}{k_{s} r}\right)+n(n+1)\left(\frac{k_{p}}{k_{s}}\right)^{3} \\
& \left.\times\left(\mathbf{L}_{m n}^{i}(\mathbf{r})+a_{m n}^{3} \mathbf{L}_{m n}^{e}(\mathbf{r})+\frac{a_{m n}^{4}}{n(n+1)}\left(\frac{k_{s}}{k_{p}}\right)^{3} \mathbf{N}_{m n}^{e}(\mathbf{r})\right) \frac{f_{n}^{e}\left(k_{p} r\right)}{k_{p} r}\right] R^{2}
\end{aligned}
$$




$$
\mathbf{V}_{m n}^{c}(\mathbf{r})=\frac{2 i k_{s}}{\mu \sqrt{n(n+1)}}\left[\mathbf{M}_{m n}^{i}(\mathbf{r})+a_{m n}^{1} \mathbf{M}_{m n}^{e}(\mathbf{r})\right] f_{n}^{e}\left(k_{p} r\right) R^{2} \text {. }
$$

The above relations (4.5)-(4.7) hold for $r=|\mathbf{r}|<R$.

In what follows we will examine the case of the rigid sphere. So, in order to find the eigenvalues of $M$, the following relation has to be satisfied:

$$
M \mathbf{u}(\mathbf{r})=\lambda \mathbf{u}(\mathbf{r}), \quad \mathbf{r} \in \partial D_{R} .
$$

Taking into account that $\left\{\mathbf{P}_{m n}, \mathbf{B}_{m n}, \mathbf{C}_{m n}\right\}$ is a basis in $\left(L_{2}(\partial D)\right)^{3}$, we can express $\mathbf{u}(\mathbf{r})$ as a linear combination of the surface vector spherical harmonics. So

$$
\mathbf{u}(\mathbf{r})=\sum_{n=0,1,1}^{\infty} \sum_{m=-n}^{n}\left[c_{m n}^{1} \mathbf{P}_{m n}(\theta, \phi)+c_{m n}^{2} \mathbf{B}_{m n}(\theta, \phi)+c_{m n}^{3} \mathbf{C}_{m n}(\theta, \phi)\right],
$$

where $\mathbf{r} \in \partial D_{R}$. From (4.8)-(4.9), taking into account (A.1)-(A.3), we arrive at the relation

$$
\begin{aligned}
\sum_{n=0,1,1}^{\infty} & \sum_{m=-n}^{n}\left[4 i k_{s}^{2} R^{2}\left(A_{m n}^{1} c_{m n}^{1}+\sqrt{n(n+1)} A_{m n}^{3} c_{m n}^{2}\right) \mathbf{P}_{m n}(\theta, \phi)\right. \\
& +2 i k_{s}^{2} R^{2}\left(\sqrt{n(n+1)} A_{m n}^{2} c_{m n}^{1}+A_{m n}^{4} c_{m n}^{2}\right) \mathbf{B}_{m n}(\theta, \phi) \\
& \left.+2 i k_{s}^{3} R^{3}\left(F_{n_{1}}^{e}\left(k_{s} R\right)+a_{m n}^{1} F_{n_{1}}^{e}\left(k_{s} R\right) f_{n}^{e}\left(k_{s} R\right) c_{m n}^{3} \mathbf{C}_{m n}(\theta, \phi)\right)\right] \\
= & \lambda \sum_{n=0,1.1}^{\infty} \sum_{m=-n}^{n}\left[c_{m n}^{1} \mathbf{P}_{m n}(\theta, \phi)+c_{m n}^{2} \mathbf{B}_{m n}(\theta, \phi)+c_{m n}^{3} \mathbf{C}_{m n}(\theta, \phi)\right]
\end{aligned}
$$

where the $A$ 's are given by (A.4)-(A.7). Forming the inner products of (4.10) with the surface vector spherical harmonics and taking into account orthogonality relations we arrive at the homogeneous system

$$
\begin{aligned}
\left(\lambda-4 i k_{s}^{2} R^{2} A_{m n}^{1}\right) c_{m n}^{1}-4 i k_{s}^{2} R^{2} \sqrt{n(n+1)} A_{m n}^{3} c_{m n}^{2}=0, \\
2 i k_{s}^{2} R^{2} \sqrt{n(n+1)} A_{m n}^{2} c_{m n}^{1}+\left(\lambda-2 i k_{s}^{2} R^{2} A_{m n}^{4}\right) c_{m n}^{2}=0, \\
{\left[\lambda-2 i k_{s}^{3} R^{3}\left(F_{n_{1}}^{i}\left(k_{s} R\right)+a_{m n}^{1} F_{n_{1}}^{e}\left(k_{s} R\right)\right) f_{n}^{e}\left(k_{s} R\right)\right] c_{m n}^{3}=0 . }
\end{aligned}
$$

In order that the solution of the above system be non-trivial, its determinant must vanish. So we arrive at the following relations which the eigenvalues must satisfy:

$$
\lambda=2 i k_{s}^{3} R^{3}\left(F_{n_{1}}^{i}\left(k_{s} R\right)+a_{m n}^{1} F_{n_{1}}^{e}\left(k_{s} R\right)\right) f_{n}^{e}\left(k_{s} R\right)
$$

or

$$
\lambda^{2}-2 i k_{s}^{2} R^{2}\left(A_{m n}^{4}+2 A_{m n}^{1}\right) \lambda-8 k_{s}^{4} R^{4} A_{m n}^{1} A_{m n}^{4}+8 k_{s}^{4} R^{4} n(n+1) A_{m n}^{2} A_{m n}^{3}=0 .
$$


From (4.11)-(4.12) we can find the eigenvalues $\lambda_{m n}^{M}$ of $M^{*} M$. So, from (4.11),

$$
\lambda_{m n}^{M}=4 k_{s}^{6} R^{6}\left|F_{n_{1}}^{i}\left(k_{s} R\right)+a_{m n}^{1} F_{n_{1}}^{e}\left(k_{s} R\right)\right|^{2}\left|f_{n}^{e}\left(k_{s} R\right)\right|^{2} .
$$

Obviously, in order to minimise the condition number we have to choose the coefficients $a_{m n}^{i}, i=1, \ldots, 5$, in such a way that all eigenvalues become 1 . Then the condition number is 1 . Hence after some computational effort we conclude that the optimal choice is

$$
a_{m n}^{1}=\frac{e^{i\left(\phi_{m n}-3 \pi / 2\right)}}{2}\left[\frac{f_{n}^{i}\left(k_{s} R\right)}{f_{n}^{e}\left(k_{s} R\right)}-\frac{F_{n_{1}}^{i}\left(k_{s} R\right)}{F_{n_{1}}^{e}\left(k_{s} R\right)}\right]-\frac{F_{n_{1}}^{i}\left(k_{s} R\right)}{F_{n_{1}}^{e}\left(k_{s} R\right)},
$$

where $\phi_{m n}$ are arbitrary real numbers. While any real $\phi_{m n}$ will suffice to minimise the condition number there is one choice which will simultaneously minimise the norm of the integral operator $M$. So, for $\phi_{m n}=-\pi / 2$, we take

$$
a_{m n}^{1}=-\frac{1}{2}\left[\frac{f_{n}^{i}\left(k_{s} R\right)}{f_{n}^{e}\left(k_{s} R\right)}+\frac{F_{n_{1}}^{i}\left(k_{s} R\right)}{F_{n_{1}}^{e}\left(k_{s} R\right)}\right] .
$$

These are the same coefficients which we found in [1] where we established the criterion of minimisation of the operator norm. From (4.12), using similar arguments as previously, in order that all the eigenvalues be equal to 1 , we obtain the relations

$$
\begin{aligned}
8 k_{s}^{4} R^{4}\left[n(n+1) A_{m n}^{2} A_{m n}^{3}-A_{m n}^{1} A_{m n}^{4}\right] & =e^{2 i \theta_{m n}}, \\
-2 i k_{s}^{2} R^{2}\left[A_{m n}^{4}+2 A_{m n}^{1}\right] & =\rho_{m n} e^{i \theta_{m n}},
\end{aligned}
$$

where $\rho_{m n}, \theta_{m n}$ are arbitrary real numbers satisfying the inequalities $0 \leq \rho_{m n} \leq 2$, $0 \leq \theta_{m n}<2 \pi$. Obviously there are infinitely many choices of coefficients $a_{m n}^{i}$, $i=2,3,4,5$, which satisfy the imposed conditions. If we choose as $a_{m n}^{i}$ the coefficients which minimise the norm of the operator [1], after some calculations we obtain $A_{m n}^{1}=A_{m n}^{4} / 2=-i /\left(4 k_{s}^{2} R^{2}\right), A_{m n}^{2}=A_{m n}^{3}=0$. For these values of $A$ 's and for $\rho_{m n}=2, \theta_{m n}=\pi$, we find that (4.12) has a double root $\lambda=1$.

The above choice of coefficients does not satisfy the inequalities imposed on the coefficients by the uniqueness theorem in $[2,7]$. But, as in [1], it has been proved that with this choice, the norm of the modified integral operator equals zero. So the boundary integral equation is uniquely solvable.

Following the same motivation as previously we can handle Neumann's problem. Proceeding in this way, we obtain a result similar to (4.11) which must be satisfied by the eigenvalues of $N$. In view of this we take the relation for the eigenvalues of $N^{*} N$

$$
\lambda_{m n}^{N}=4 k_{s}^{6} R^{6}\left|f_{n}^{i}\left(k_{s} R\right)+a_{m n}^{1} f_{n}^{e}\left(k_{s} R\right)\right|^{2}\left|F_{n_{1}}^{e}\left(k_{s} R\right)\right|^{2},
$$




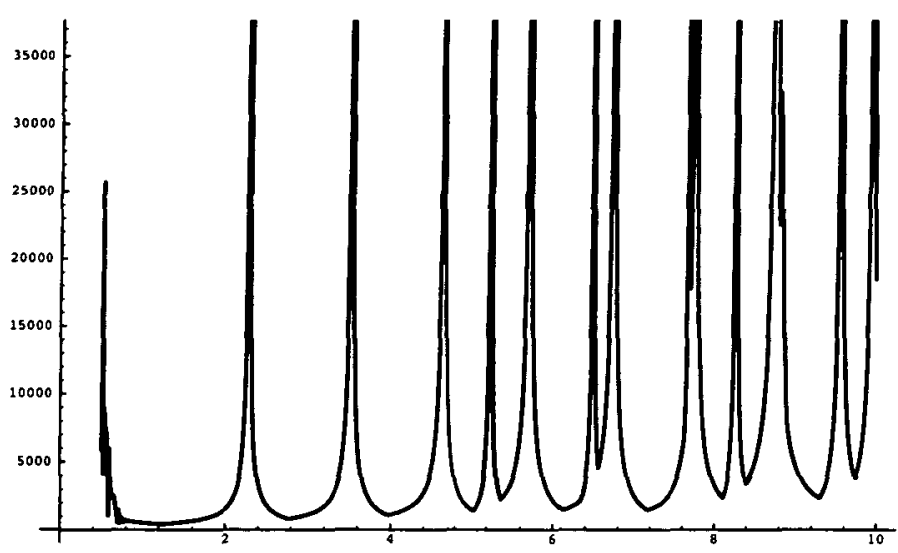

FIGURE 1. Condition number $\operatorname{cond}(M)$ versus $k_{s} R$ : Unmodified case.

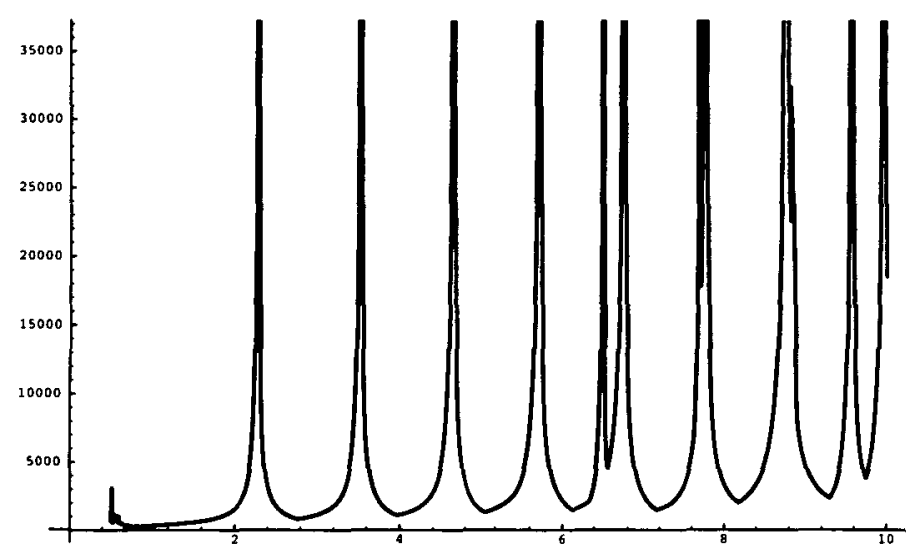

FIGURE 2. Condition number cond $(M)$ versus $k_{s} R$ : Regular part with the first two spheroidal terms.

from which, imposing the condition $\lambda^{N}=1$, we take

$$
a_{m n}^{1}=\frac{e^{i \phi_{m n}}}{2 k_{s}^{3} R^{3} f_{n}^{e}\left(k_{s} R\right) F_{n_{1}}^{e}\left(k_{s} R\right)}-\frac{f_{n}^{i}\left(k_{s} R\right)}{f_{n}^{e}\left(k_{s} R\right)}, \quad \phi_{m n} \in[0,2 \pi),
$$

where $\phi_{m n}$ is an arbitrary real number. For $\phi_{m n}=\pi / 2$ we take

$$
a_{m n}^{1}=-\frac{1}{2}\left[\frac{f_{n}^{i}\left(k_{s} R\right)}{f_{n}^{e}\left(k_{s} R\right)}+\frac{F_{n_{1}}^{i}\left(k_{s} R\right)}{F_{n_{1}}^{e}\left(k_{s} R\right)}\right],
$$

that is, the same coefficient that minimises the norm of the operator $N$. The corresponding relation to (4.12) for the case of the cavity gives, for the eigenvalues $\lambda_{m n}^{N}$, 


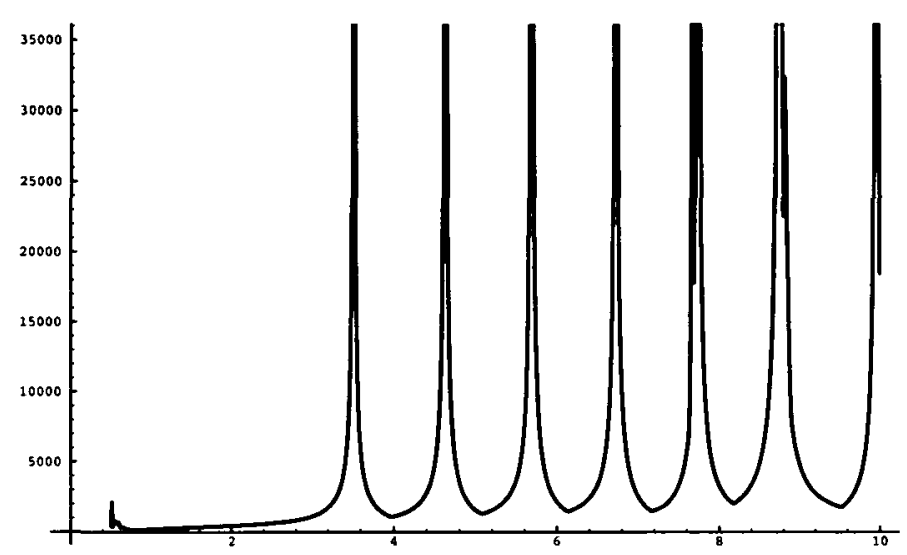

FIGURE 3. Condition number cond $(M)$ versus $k_{s} R$ : Regular part with the first three spheroidal terms.

the relations

$$
\begin{gathered}
4 k_{s}^{4} R^{4}\left[2 n(n+1) A_{m n}^{2} A_{m n}^{3}+\frac{1}{k_{s}^{4} R^{4}}-\frac{i}{k_{s}^{2} R^{2}}\left(2 A_{m n}^{1}+A_{m n}^{4}\right)-2 A_{m n}^{1} A_{m n}^{4}\right]=e^{2 i \theta_{m n}}, \\
2 i k_{s}^{2} R^{2}\left[\frac{2 i}{k_{s}^{2} R^{2}}+2 A_{m n}^{1}+A_{m n}^{4}\right]=\rho_{m n} e^{i \theta_{m n}}
\end{gathered}
$$

where $\rho_{m n}, \theta_{m n}$ are arbitrary real numbers satisfying the inequalities $0 \leq \rho_{m n} \leq 2$, $0 \leq \theta_{m n}<2 \pi$. As previously, the coefficients which minimise the operator norm also minimise the condition number with $\rho_{m n}=2, \theta_{m n}=\pi$. So the criterion for the minimisation of the condition number for the two exterior problems is established.

\section{The perturbation of the sphere}

As in $[2,9,11]$ we can consider a family of non-spherical boundaries given parametrically by the relation $r_{\epsilon}=R+\epsilon \psi(\theta, \phi), 0 \leq \theta \leq \pi, 0 \leq \phi \leq 2 \pi$, where $\psi$, $\partial \psi / \partial \theta,(1 / \sin \theta)(\partial \psi / \partial \phi)$ are all bounded. We will use the estimates for the Hansen vectors given in [1],

$$
\mathbf{M}_{m n}^{e}\left(\mathbf{r}_{\epsilon}\right)=\mathbf{M}_{m n}^{e}\left(\mathbf{r}_{o}\right)+O(\epsilon), \quad T \mathbf{M}_{m n}^{e}\left(\mathbf{r}_{\epsilon}\right)=T \mathbf{M}_{m n}^{e}\left(\mathbf{r}_{o}\right)+O(\epsilon),
$$

where $\mathbf{r}_{\epsilon}$ is a point in the perturbed surface while $\mathbf{r}_{o}$ describes points on the sphere. Similar estimates hold for $\mathbf{N}_{m n}^{e}$ and $\mathbf{L}_{m n}^{e}$. In [1] it has been proved that the boundary integral operator is a perturbation of the boundary integral operator defined on the sphere. In view of these estimates it is straightforward that the eigenvalues of the 


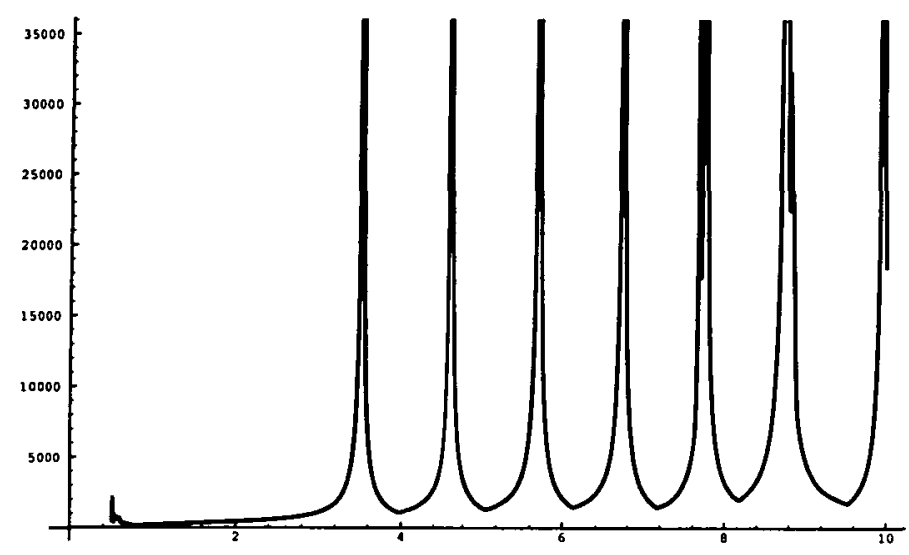

FIGURE 4. Condition number cond $(M)$ versus $k_{s} R$ : Regular part with the first three toroidal and three spheroidal terms.

perturbed $M$ and $N$ are perturbations of the eigenvalues of the original operators. So

$$
\operatorname{cond}(M)=1+O(\epsilon)
$$

Similarly for $N$. In view of this relation the choice proposed for the sphere is also, in general, good enough for the perturbed spherical domains.

\section{Numerical results}

In this section we will examine numerically the case of the rigid sphere. As already mentioned, the homogeneous integral equation corresponding to (2.5) is uniquely solvable unless the adjoint interior Neumann problem has non-trivial solutions. The investigation of this problem has been done in [3] by considering the oscillations of a homogeneous sphere. Two types of eigenvalues and corresponding eigenfrequencies exist. The first type are the toroidal oscillations which are expressed via the $\mathbf{M}_{m n}$ eigenvectors. The second type of eigenvectors are known as spheroidal oscillations and are expressed via the $\mathbf{N}_{m n}$ and $\mathbf{L}_{m n}$ eigenvectors.

When we plot the condition number for the original integral operator versus $k_{s} R$, for an elastic medium with $k_{p}=1.1 k_{s}$, then ill-conditioned behaviour is observed at the values of the toroidal and the spheroidal eigenvalues. In the plotted figures of the condition number versus $k_{s} R$, the existence of eigenfrequencies is reflected in the existence of points where the values of the condition number is extremely increased. We can see the unmodified case in Figure 1. In Figure 2 we show the effect of considering the regular part of the modification with two spheroidal terms. We see that 


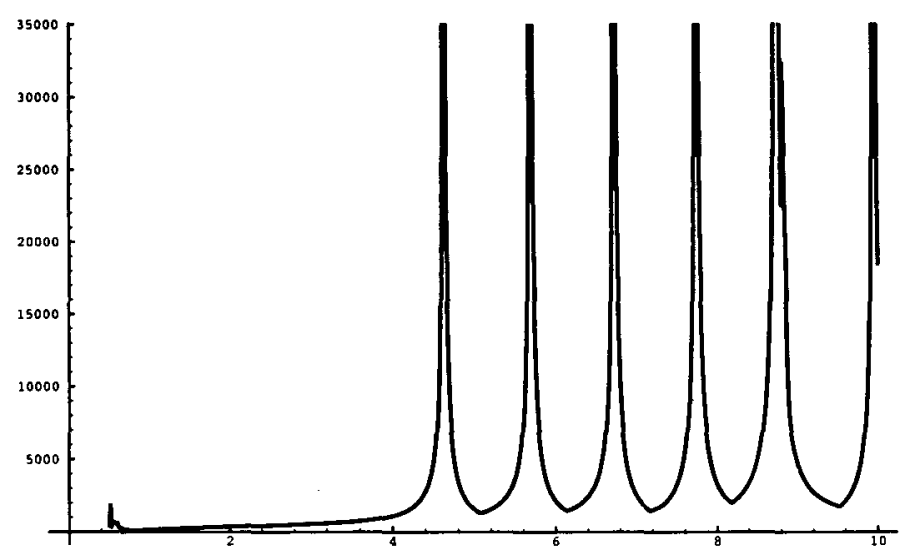

FIGURE 5. Condition number $\operatorname{cond}(M)$ versus $k_{s} R$ : Regular part with the first four spheroidal and four toroidal terms.

the eigenfrequencies due to the first spheroidal oscillations are removed. In Figure 3 we observe the effect of adding three spheroidal terms in the modification. In Figure 4 the addition of three spheroidal and three toroidal terms removes all the eigenvalues in the range $0<k_{s} R<3.5$. In Figure 5 the effect of the consideration of four spheroidal and four toroidal terms is to completely remove all the eigenfrequencies in the range $0<k_{s} R<4.5$. Similarly in Figure 6, considering that the regular part consists of five spheroidal and five toroidal terms, we completely remove all the eigenfrequencies in the range $0<k_{s} R<5.5$. So the introduction of the modified terms enlarges the region of unique solvability of the boundary integral equation.

From the above numerical consideration and relation (5.1) it is also deduced that when we have to solve numerically the modified integral equation for shapes which are small perturbations of a sphere the terms of the modification required in the representation of the Green's function depend on the values of $k_{s} R$ analogously as for the spherical case.

\section{Appendix A.}

Now we will produce, with the aid of the potential theoretic jump relations, boundary integral equations on the surface of a rigid sphere and of a spherical cavity, with densities being the vector spherical harmonics.

For the rigid sphere with radius $R$ we conclude that the following boundary integral relations hold:

$$
\left(I+\bar{K}_{1}^{*}\right) \mathbf{P}_{m n}(\theta, \phi)=2 i k_{s}^{2} R^{2}\left(2 A_{m n}^{1} \mathbf{P}_{m n}(\theta, \phi)+\sqrt{n(n+1)} A_{m n}^{2} \mathbf{B}_{m n}(\theta, \phi)\right),
$$




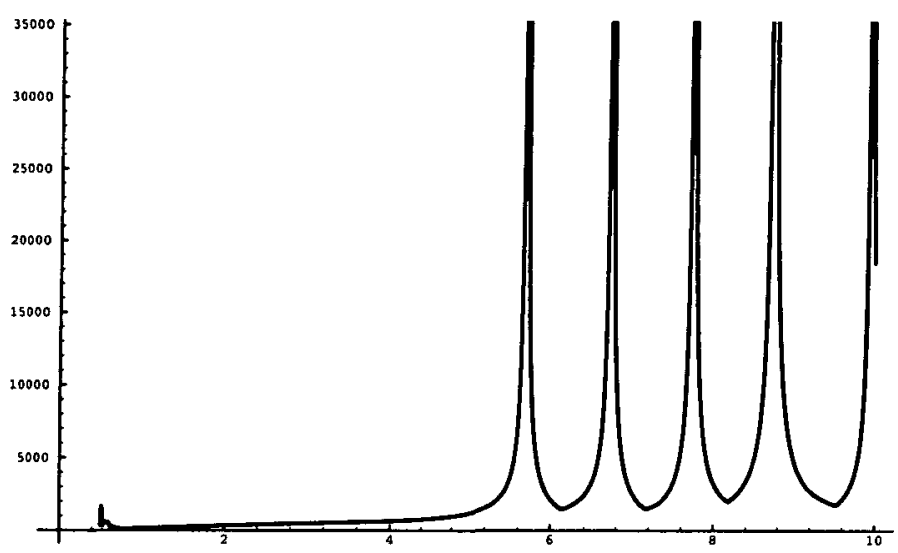

FIGURE 6. Condition number $\operatorname{cond}(M)$ versus $k_{s} R$ : Regular part with the first five spheroidal and five toroidal terms.

$$
\begin{aligned}
& \left(I+\bar{K}_{1}^{*}\right) \mathbf{B}_{m n}(\theta, \phi)=2 i k_{s}^{2} R^{2}\left(2 \sqrt{n(n+1)} A_{m n}^{3} \mathbf{P}_{m n}(\theta, \phi)+A_{m n}^{4} \mathbf{B}_{m n}(\theta, \phi)\right) \\
& \left(I+\bar{K}_{1}^{*}\right) \mathbf{C}_{m n}(\theta, \phi)=2 i k_{s}^{3} R^{3}\left(F_{n_{1}}^{i}\left(k_{s} R\right)+a_{m n}^{1} F_{n_{1}}^{e}\left(k_{s} R\right)+f_{n}^{e}\left(k_{s} R\right)\right) \mathbf{C}_{m n}(\theta, \phi)
\end{aligned}
$$

where

$$
\begin{aligned}
& A_{m n}^{1}=n(n+1) b_{m n}^{1} \frac{f_{n}^{e}\left(k_{s} R\right)}{k_{s} R}+\left(\frac{k_{p}}{k_{s}}\right)^{4} b_{m n}^{2} f_{n}^{e \prime}\left(k_{p} R\right), \\
& A_{m n}^{2}=b_{m n}^{3} \frac{f_{n}^{e}\left(k_{s} R\right)}{k_{s} R}+\left(\frac{k_{p}}{k_{s}}\right)^{4} b_{m n}^{4} f_{n}^{e^{\prime}}\left(k_{p} R\right), \\
& A_{m n}^{3}=b_{m n}^{1}\left(f_{n}^{e \prime}\left(k_{s} R\right)+\frac{f_{n}^{e}\left(k_{s} R\right)}{k_{s} R}\right)+\left(\frac{k_{p}}{k_{s}}\right)^{4} b_{m n}^{2} \frac{f_{n}^{e}\left(k_{p} R\right)}{k_{p} R} \\
& A_{m n}^{4}=b_{m n}^{3}\left(f_{n}^{e \prime}\left(k_{s} R\right)+\frac{f_{n}^{e}\left(k_{s} R\right)}{k_{s} R}\right)+n(n+1)\left(\frac{k_{p}}{k_{s}}\right)^{4} b_{m n}^{4} \frac{f_{n}^{e}\left(k_{p} R\right)}{k_{p} R} .
\end{aligned}
$$

The quantities $b_{m n}^{i}$ in (A.4)-(A.7) are

$$
\begin{aligned}
& b_{m n}^{1}=F_{n_{1}}^{i}\left(k_{s} R\right)+a_{m n}^{2} F_{n_{1}}^{e}\left(k_{s} R\right)+\frac{k_{p}}{k_{s}} \frac{a_{m n}^{5}}{n(n+1)} F_{n_{3}}^{e}\left(k_{p} R\right), \\
& b_{m n}^{2}=F_{n_{3}}^{i}\left(k_{p} R\right)+a_{m n}^{3} F_{n_{3}}^{e}\left(k_{p} R\right)+\left(\frac{k_{s}}{k_{p}}\right)^{4} a_{m n}^{4} F_{n_{1}}^{e}\left(k_{p} R\right), \\
& b_{m n}^{3}=F_{n 2}^{i}\left(k_{s} R\right)+a_{m n}^{2} F_{n_{2}}\left(k_{s} R\right)+2 \frac{k_{p}}{k_{s}} a_{m n}^{5} F_{n_{1}}^{e}\left(k_{p} R\right), \\
& b_{m n}^{4}=2 F_{n_{1}}^{i}\left(k_{p} R\right)+2 a_{m n}^{3} F_{n_{1}}^{e}\left(k_{p} R\right)+\left(\frac{k_{s}}{k_{p}}\right)^{4} \frac{a_{m n}^{4}}{n(n+1)} F_{n_{2}}^{e}\left(k_{s} R\right),
\end{aligned}
$$


with [3]

$$
\begin{aligned}
& F_{n_{1}}^{e, i}(z)=\frac{d}{d z}\left(\frac{1}{z} f_{n}^{e, i}(z)\right), \\
& F_{n_{2}}^{e, i}(z)=\frac{d^{2}}{d z^{2}}\left(f_{n}^{e, i}(z)\right)+\frac{(n-1)(n+2)}{z^{2}} f_{n}^{e, i}(z), \\
& F_{n_{3}}^{e, i}(z)=\frac{d^{2}}{d z^{2}}\left(f_{n}^{e, i}(z)\right)+\frac{\lambda}{2 \mu} f_{n}^{e, i}(z) .
\end{aligned}
$$

For the spherical cavity with radius $R$, after some computation we conclude that

$$
\begin{aligned}
\left(I-K_{1}\right) \mathbf{P}_{m n}(\theta, \phi)= & -2 i k_{s}^{2} R^{2}\left(\left(\frac{i}{k_{s}^{2} R^{2}}+2 A_{m n}^{1}\right) \mathbf{P}_{m n}(\theta, \phi)\right. \\
& \left.+\sqrt{n(n+1)} A_{m n}^{2} \mathbf{B}_{m n}(\theta, \phi)\right), \\
\left(I-K_{1}\right) \mathbf{B}_{m n}(\theta, \phi)= & -2 i k_{s}^{2} R^{2}\left(2 \sqrt{n(n+1)} A_{m n}^{3} \mathbf{P}_{m n}(\theta, \phi)\right. \\
& \left.+\left(\frac{i}{k_{s}^{2} R^{2}}+2 A_{m n}^{4}\right) \mathbf{B}_{m n}(\theta, \phi)\right), \\
\left(I-K_{1}\right) \mathbf{C}_{m n}(\theta, \phi)= & -2 i k_{s}^{3} R^{3}\left(f_{n}^{i}\left(k_{s} R\right)+a_{m n}^{1} f_{n}^{e}\left(k_{s} R\right)\right) F_{n_{1}}^{e}\left(k_{s} R\right) \mathbf{C}_{m n}(\theta, \phi),
\end{aligned}
$$

where the $A$ 's appearing are given by (A.5)-(A.7).

\section{References}

[1] E. Argyropoulos and K. Kiriaki, "A criterion of optimization of the modified Green's function in linear elasticity", Internat. J. Engrg. Sci. 37 (1999) 1441-1460.

[2] E. Argyropoulos, K. Kiriaki and G. F. Roach, "A modified Green's function technique for the exterior Dirichlet problem in linear elasticity", Q. J. Mech. Appl. Math. 52 (1998) 275-295.

[3] B.-M. Ari and S. J. Singh, Seismic waves and sources (Springer, 1981).

[4] L. Bencheikh, "Modified fundamental solutions for the scattering of elastic waves by a cavity", $Q$. J. Mech. Appl. Math. 43 (1990) 58-73.

[5] L. Bencheikh, "Modified fundamental solutions for the scattering of elastic waves by a cavity: Numerical results", Internat. J. Numer. Methods Engrg. 36 (1993) 3283-3302.

[6] L. Collatz, Funktionalanalysis und numerische Mathematik (Springer, Berlin, 1964).

[7] D. Gintides, K. Kiriaki and G. F. Roach, "Modified Green's function techniques for exterior problems in elasticity", Math. research report no. 5, Univ. Strathclyde, 1993.

[8] D. S. Jones, "Integral equations for the exterior acoustic problem", Q. J. Mech. Appl. Math. 27 (1974) 129-142.

[9] R. E. Kleinman and R. Kress, "On the condition number of integral equations in acoustics using modified fundamental solutions", IMA J. Appl. Math. 31 (1983) 79-90.

[10] R. E. Kleinman and G. F. Roach, "On modified Green functions in exterior problems for the Helmholtz equation”, Proc. Roy. Soc. London A 383 (1982) 313-332. 
[11] R. E. Kleinman and G. F. Roach, "Operators of minimal norm via modified Green's functions", Proc. Roy. Soc. Edinburgh 94A (1983) 163-178.

[12] V. D. Kupradze, T. G. Gegelia, M. O. Basheleĭshvili and T. V. Burchuladze, Three-dimensional problems of the mathematical theory of elasticity and thermoelasticity, North-Holland Series in Appl. Math. and Mech. 25., Translated from the second Russian edition, Edited by V. D. Kupradze (North-Holland, Amsterdam, 1979).

[13] F. Ursell, "On exterior problems of acoustics II", Math. Proc. Cambridge Philos. Soc. 84 (1978) 545-548. 